\title{
Anxiety and autonomic response to social-affective stimuli in individuals with Williams syndrome
}

\author{
Rowena Ng1,2, Ursula Bellugi ${ }^{1}$, \& Anna Järvinen ${ }^{1 *}$ \\ ${ }^{1}$ Laboratory for Cognitive Neuroscience, the Salk Institute for Biological Studies, La Jolla, CA, \\ USA \\ ${ }^{2}$ University of Minnesota, Twin Cities; Institute of Child Development, Minneapolis, MN, USA
}

\section{Correspondence:}

Anna Järvinen, PhD

Laboratory for Cognitive Neuroscience

The Salk Institute for Biological Studies

10010 North Torrey Pines Road

La Jolla, CA 92037-1002

Email: pasley@salk.edu 
Anxiety and autonomic response to emotion in WS

\begin{abstract}
Background: Williams syndrome (WS) is a genetic condition characterized by an unusual "hypersocial" personality juxtaposed by high anxiety. Recent evidence suggests that autonomic reactivity to affective face stimuli is disorganised in WS, which may contribute to emotion dysregulation and/or social disinhibition. Methods: Electrodermal activity (EDA) and mean interbeat interval (IBI) of 25 participants with WS (19 - 57 years old) and 16 typically developing (TD; 17 - 43 years old) adults were measured during a passive presentation of affective face and voice stimuli. The Beck Anxiety Inventory was administered to examine associations between autonomic reactivity to social-affective stimuli and anxiety symptomatology. Results: The WS group was characterized by higher overall anxiety symptomatology, and poorer anger recognition in social visual and aural stimuli relative to the TD group. No between-group differences emerged in autonomic response patterns. Notably, for participants with WS, increased anxiety was uniquely associated with diminished arousal to angry faces and voices. In contrast, for the TD group, no associations emerged between anxiety and physiological responsivity to social-emotional stimuli. Conclusions: The anxiety associated with WS appears to be intimately related to reduced autonomic arousal to angry social stimuli, which may also be linked to the characteristic social disinhibition.
\end{abstract}

Keywords: Williams syndrome, autonomic nervous system, electrodermal activity, anxiety, social behaviour, emotion, stress regulation 
Anxiety and autonomic response to emotion in WS

What this paper adds: Research have suggested that those with WS show more anxiety yet less social inhibition than TD or developmentally delayed individuals. However, to date, the association between anxiety and autonomic arousal to social affective stimuli, which serves as an internal cue to activate individuals' fight/flight and approach/avoidance response, remains unclear. This question is of particular importance, as abnormal physiological reactivity to specific social-affective stimuli may underpin the hypersociability that characterizes WS. To address this gap in literature, autonomic reactivity to scary, happy, and angry social stimuli and caregiver/self-reported ratings of anxiety in TD and WS individuals. Results implicate diminished arousal towards fearful social stimuli is related to increased anxiety in those with WS uniquely. Further research targeting psychophysiological responsivity to social-affective stimuli in WS, preferably in naturalistic interactive settings, is warranted. 
Anxiety and autonomic response to emotion in WS

\section{Introduction}

Williams syndrome (WS) is a neurodevelopmental disorder stemming from the deletion of approximately 25 genes on chromosome 7q11.23 (Ewart et al., 1993; Hillier et al., 2003). Cognitively, individuals with WS function at the level associated with mild-to-moderate intellectual impairment (Searcy et al., 2004). Affected individuals exhibit an unusual "hypersocial” profile, including increased willingness to approach strangers (Järvinen-Pasley et al., 2008, 2013, 2015; Little et al., 2013; Martens et al., 2009, 2012; Frigerio et al., 2006), atypically expressive language used maximally for social engagement (Järvinen-Pasley et al., 2008), and heightened attention toward faces (see Järvinen-Pasley et al., 2008; Riby \& Hancock, 2008, 2009). Specific personality characteristics that distinguish individuals with WS from persons with other learning difficulties conditions include a lack of shyness, increased empathy, gregariousness, and elevated sensitivity/anxiety (Klein-Tasman \& Mervis, 2003).

Substantial evidence indicates that a central component of the psycho-emotional phenotype of WS encompasses elevated phobic fear and anxiety (Dodd et al., 2009; Dykens, 2003; Leyfer et al., 2006, 2009). Individuals with WS across the life-span demonstrate significantly increased prevalence for anxiety as compared to the typically developing (TD) population (Leyfer et al., 2006; Ng et al., 2014; Rodgers et al., 2012; Sarimski, 1997; WoodruffBorden et al., 2010), and higher anxiety relative to other developmental disabilities (DD) (Dimitropoulos et al., 2009; Dykens et al., 2005). The most prevalent diagnoses pertain to specific phobia, generalised anxiety disorder, separation anxiety disorder, and obsessivecompulsive disorder, respectively (Dodd et al., 2009; Dykens, 2003; Leyfer et al., 2006); however, the underlying mechanisms remain unclear. Associations Between Anxiety and Social-Affective Processing in Williams Syndrome 
Anxiety and autonomic response to emotion in WS

In the context of the "hypersocial" phenotype of WS, some literature has suggested that the anxiety characterising WS may be non-social in nature (Dodd et al., 2009; Dykens, 2003; Ng et al., 2014; Perez-Garcia et al., 2011; Rodgers et al., 2012). This claim is further supported by evidence that individuals with WS do not demonstrate clinical levels of social phobia (e.g., Dodd et al., 2009; Riby et al., 2014), a feature that is also aptly demonstrated by their fearless social behaviour in real life (e.g., Doyle et al., 2004; Martens et al., 2009; Riby et al., 2014). At the same time, this is not to say that social behaviour and anxiety are clearly independent in WS. A recent study employing the parent-report inventories of the Spence Children's Anxiety Scale and the Social Responsiveness Scale showed that children and adults with WS, who were classified as highly anxious, demonstrated more pronounced social dysfunction as compared to their counterparts with low anxiety (Riby et al., 2014). Additionally, Kirk et al. (2013) reported that increased anxiety in individuals with WS was associated with unique face gaze patterns (i.e., greater attention to the mouth and decreased attention to the eye region of fearful faces) and greater social dysfunction. Taken together, emerging evidence suggests that there is some level of relationship between social functioning and anxiety.

A shared feature between the sufferers of social anxiety disorder and those with WS is an atypical processing of facial emotion (Binelli et al., 2014). Individuals with WS exhibit reduced identification of negative emotions for face and voice stimuli (Plesa Skwerer et al., 2006; Järvinen-Pasley et al., 2010a, 2010b), together with a diminished sensitivity (Santos et al., 2010) and reduced attention (Dodd \& Porter, 2010) toward angry faces. Interestingly, these individuals also demonstrate "hypervigilance" toward positive emotion, as evidenced by an attentional bias toward happy face stimuli (Dodd \& Porter, 2010), near typical emotion identification ability for positively valenced stimuli (Järvinen-Pasley et al., 2010a, 2010b; Plesa Skwerer et al., 2006), 
Anxiety and autonomic response to emotion in WS

more positive evaluation of unapproachable-looking unfamiliar faces as compared to TD peers (Bellugi et al., 1999; Martens et al., 2009), and a tendency toward perceiving happy faces as unusually approachable (Porter et al., 2007).

The uneven behavioural processing of positive and negative affect in WS is also evident at the neural level (Meyer-Lindenberg et al., 2005b; Haas et al., 2009). Notably, such individuals show augmented amygdala response to non-social fearful stimuli, lending credence to the postulation that their anxiety is non-social in nature (Meyer-Lindenberg et al., 2005a; ThorntonWells et al., 2011). It is thus possible that the increased subjective/psychological anxiety in WS may be linked to the well-established alterations in the neural circuit subserving fear/threat processing, most notably the amygdala (Haas et al., 2009, 2012; Martens et al., 2009; MeyerLindenberg et al., 2005a), which is at the heart of the neural circuitry responsible for the processing of socially and emotionally relevant information (e.g., Adolphs, 2003).

\subsection{Autonomic functioning and Williams syndrome}

Recent evidence indicates that individuals with WS exhibit atypical autonomic nervous system (ANS) functioning in response to emotional stimuli (Järvinen et al., 2012, 2015; Plesa Skwerer et al., 2009; Riby et al., 2012; for a review, see Järvinen \& Bellugi, 2013). Autonomic reactivity, as indexed by measures such as electrodermal (EDA) responses and heart rate, reflect sympathetic activation for fight-or-flight behaviours when in fear, and/or parasympathetic activity for resting responses when relaxed (Herman et al., 2005). These physiological cues are part of a central stress system that is regulated by input and output from the hippocampus, amygdala, and prefrontal cortex (Herman et al. 2005), regions that have been found to function atypically in those with WS (Hass et al., 2009, 2010; Meyer-Lindenberg et al., 2005a, 2005b; Paul et al., 2009). As such, there has been a growing interest in characterizing the role of ANS 
Anxiety and autonomic response to emotion in WS

dysfunction in social-affective perception and interpersonal behaviours in these individuals. Importantly, anxiety (without a WS diagnosis) has been linked to ANS dysfunction, including decreased parasympathetic activity (Beachaine, 2001) and hyperactivation of sympathetic nervous system (Friedman, 2007). To this end, given the recent evidence linking anxiety with interpersonal functioning (e.g., Kirk et al., 2013), elucidating the psychophysiological correlates of anxiety may be a valuable avenue toward understanding the interplay between the social and anxiety phenotypes of WS.

Recent evidence has begun to implicate ANS dysfunction in altered social-emotional perception in individuals with WS. Plesa Skwerer and coauthors (2009) documented hypoarousal to dynamic face expressions in adolescents and adults with WS, when compared to TD and DD controls matched on chronological age (CA). Additionally, Järvinen-Pasley et al. (2012) found that participants with WS showed a lack of habituation to happy faces. Based on these observations, it was hypothesised that individuals with WS are more likely to approach others, as they lack the typical familiarisation effect that one acquires when meeting the same individual over time (see Järvinen-Pasley et al., 2012). In another study, adults with WS exhibited the highest relative EDA reactivity to happy and lowest arousal to fearful faces, whereas, TD participants demonstrated the contrasting pattern (Järvinen et al., 2015). In adults with WS, increased EDA reactivity to negative faces was also related to the approachability perception toward the same face stimuli (Järvinen et al., 2015). Taken together, the existing sparse evidence on autonomic responses to social emotional stimuli suggests disorganisation of the ANS in WS, which may underlie the unusual interpersonal behaviours. Specifically, the autonomic responsivity towards positive versus negative affective stimuli in WS individuals may not provide the same negative feedback like TD adults to inform social behaviours (e.g., under 
Anxiety and autonomic response to emotion in WS

normative development, sympathetic arousal towards scary stimuli may elicit a flight response such as avoidance; whereby, among those with WS, increased arousal to such stimuli triggers more social engagement and affiliation). However, it should be noted that Riby et al. (2012) examined baseline EDA in response to live and video-mediated displays of happy, sad, and neutral affect in individuals with WS and TD controls matched for CA, and found that only live faces increased the level of arousal for those with WS and TD, suggesting that the lack of naturalistic features of social stimuli may have a role in the observed results in the above described investigations. Nevertheless, recent literature links irregularities of ANS functioning with features of the social phenotype of WS.

To our knowledge, there are no previous investigations directly exploring how anxiety may be linked to autonomic sensitivity toward social-affective information in individuals with WS. This is of clinical and theoretical importance, as the psychophysiological characteristics of individuals with WS may vitally contribute to the anxiety phenotype, social-emotional processing, and social fearlessness. For example, it is possible that ANS dysfunction for negative social stimuli (e.g., reduced sympathetic activation for faces or vocalisations) result in the absence of the fight-or-flight response, which in turn underlie the characteristic social disinhibition.

\subsection{Current study}

The purpose of the current study was to examine whether autonomic reactivity to socialaffective stimuli in individuals with WS is related to anxiety. More specifically, one aim was to elucidate the imbalanced processing of negative and positive emotion in such individuals. First, across the WS and TD groups, anxiety was measured by the Beck Anxiety Inventory (BAI; Beck, Epstein, Brown, \& Steer, 1988). Second, autonomic arousal (electrodermal response, 
Anxiety and autonomic response to emotion in WS

electrocardiogram) was measured while participants passively attended to face/voice stimuli matched for emotion (angry, fearful, happy). Finally, participants were administered a complementary active emotion-recognition task to determine their ability to identify affect in social stimuli.

We hypothesised the following: 1.) Consistent with previous evidence (Ng et al., 2014), compared to TD individuals, participants with WS would endorse more intense anxiety symptoms as reflected by higher BAI scores; 2.) Additionally, in line with the positive bias hypothesis (Frigerio et al., 2006), we predicted that the WS group would identify positive affect at a greater accuracy as compared to negative affect (angry, fearful) across both visual and vocal domains. 3.) In line with Järvinen et al. (2012), we anticipated an atypical pattern of autonomic responses to happy social stimuli. 4.) As outlined above, social affective dysfunction and anxiety may not be fully dissociable in those with WS. Accordingly, we hypothesised that the autonomic responsivity of individuals with WS to threat-related stimuli (i.e., presentations of anger and/or fear) would be related to their anxiety, which may explain the elevated anxiety and social disinhibition that this population exhibits. Finally, given the lack of literature comparing autonomic responsivity and emotion recognition across different social stimulus modalities (faces vs. voices) in WS, no a priori predictions were made regarding this condition.

\section{Methods}

\subsection{Participants}

Forty-one individuals participated in the current study: 25 individuals with WS (10 females; $M=33.45$ years), and 16 TD comparison individuals ( 11 females; $M=25.44$ years). Of note, this study was part of an extensive multisite investigation examining the link between genes, behavioural profile, cognitive and social phenotypes, and cellular mechanisms specific to 
Anxiety and autonomic response to emotion in WS

WS in contrast to normative development. Given this large-scale research effort, individuals with WS and TD were the primary recruited samples. Of note, one TD participant was 17.4 years when recruited to our multisite investigation. The subsequent data analyses were complete with and without the participants' data. Given the inclusion of the participants' data did not modify the results (i.e., within and between group differences, correlations), the data was included in the final analyses.

The genetic diagnosis of WS was established using fluorescence in situ hybridization (FISH) probes for elastin (ELN). The TD participants were also screened for history of brain trauma, psychiatric concerns, and central nervous system disorders, and were required to be native English speakers. TD individuals completed a lengthy questionnaire inquiring if they have been seen by a professional for the noted concerns, and diagnosed with traumatic brain injury, mental health ailment or neurological disorder. Caregivers of WS participants also completed an inventory to assess these areas to determine that they did not experience additional significant medical or neurological trauma in the past. None of the participants reported significant diagnostic history of anxiety or medication use related to the psychiatric condition. Visual and hearing tests were administered to ensure vision/hearing is intact or appropriately corrected. WS and TD participants also completed assessments to determine their intellectual functioning. Notably, this assessment was to ensure TD participants did not show concerns of intellectual disabilities. WS participants were administered either the Wechsler Adult Intelligence Scale Third Edition (WAIS-III; Wechsler, 1997); whereas, TD adults completed the Wechsler Abbreviated Scale of Intelligence (WASI; Wechsler, 1999).

Of note, participants were recruited as a part of a multi-site multidisciplinary program of research. However, TD were scheduled to complete all assessments within a day; whereas, those 
Anxiety and autonomic response to emotion in WS

with WS and their families were recruited to complete multi-site testing across several days. As such, due to differences in time limitations, TD participants were given the WASI over the WAIS-III. The TD participants outperformed their counterparts with WS on verbal IQ (VIQ), performance IQ (PIQ), and full-scale IQ (FSIQ) (ts > 8.34, ps <.001). Despite the betweengroup differences in cognitive functioning, such differences were not controlled for in subsequent analyses as it has been suggested controlling for IQ does not contribute to the clarity of the meaningful differences seen in the autonomic responses to valence and social content in individuals with neurodevelopmental conditions (see Cohen et al., 2013, for a discussion). Specifically, it has been postulated that IQ impairments may stem from shared ANS alterations that lead to the social-emotional processing deficits, and thus, it could be argued that socialemotional processing difficulties are not caused by IQ but rather have a shared origins rooted in the underlying neurodevelopmental disorder (Dennis et al., 2009).

All participants gave written informed consent before participation. Written informed assent was also obtained from the WS participants' parents, guardians, or conservators. Experimental procedures were approved by the Institutional Review Board at the authors' institute.

\subsection{Materials}

\subsubsection{Beck Anxiety Inventory (BAI; Beck, Epstein, Brown, \& Steer, 1988). Consistent with Ng et} al. (2014), caregivers of those with WS completed the inventory based on their interactions and observations of their child, and TD individuals were instructed to complete the inventories with a close kin or spouse for reference. Specifically, caregivers were asked "how much has your child been bothered by the following symptoms during the past month," whereas, TD adults were inquired "how much have you been bothered by the following symptoms during the past month". 
Anxiety and autonomic response to emotion in WS

This inventory consists of 21 items indexing four domains of anxiety (subjective, autonomic, neurophysiological, panic; BAI-S, BAI-A, BAI-N, BAI-P respectively), and has been empirically supported in psychometric investigations (Osman et al., 1997). Respondents were instructed to rate the degree each symptom was endorsed on a 4-point scale: 0 (Not at all), 1 (Mildly), 2 (Moderately), and 3 (Severely). Given BAI subscales comprise of differing number of items, the mean averages per sub-index were computed for statistical analysis. The total raw score (BAI-total), which is the sum of all ratings, describes the general severity of the anxiety: 07 denotes minimal anxiety, 8-15 represents mild anxiety, 16-25 refers to moderate anxiety, and 26-63 equates to severe anxiety. Importantly, psychometric investigations of the BAI and its factor structure have shown that it can be used to discriminate anxiety symptomatology in individuals with intellectual disabilities similar to the general population (Lindsay \& Skene, 2007). All participants with the exception of five individuals with WS and two TD participants completed the BAI (Beck et al., 1988). These individuals were given the BAI to complete at home given time limitations during the experimental session; however, the inventory was not returned.

2.2.3 Experimental stimuli. The visual stimuli comprised 18 standardized static images of facial expression taken from the Mac Brain / NimStim Face Stimulus Set (Tottenham et al., 2009). The faces with the highest validation ratings were selected. Similarly, the auditory stimuli comprised 18 segments of non-linguistic vocal sounds (2-3 seconds/segment) taken from the "Montreal Affective Voices", a standardized set of vocal expressions without confounding linguistic information (freely available at http://vnl.psy.gla.ac.uk/info.php?file=mav). There were 6 female and 6 male vocal and facial segments for each of three emotions (fearful, happy, angry).

\subsection{Procedure}


Anxiety and autonomic response to emotion in WS

Silver/silver chloride electrodes where applied to the skin with an isotonic sodium chloride electrolyte gel placed on the index and middle medial phalanges of the participant's left hand to record EDA, according to a standard bipolar placement (Venables \& Christie, 1980). ECG was recorded from Lead II configuration with two disposable electrodes, one attached to the right forearm and the other attached to the left ankle, below the true ankle joint. To prevent autonomic habituation effects, the psychophysiological (i.e., passive) portion of the study was administered first, followed by the active behavioral affect identification portion. The stimuli were presented on a computer running Matlab (The MathWorks Inc., Natick, MA), which delivered a digital pulse embedded in the recording at the onset of each stimulus. To measure physiological responses, following a fixation cross for $2000 \mathrm{~ms}$, each stimulus was presented for $3000 \mathrm{~ms}$, separated by an interstimulus interval (ISI) of $8000 \mathrm{~ms}$ (blank screen) for autonomic activity to return to near baseline. For the passive task, participants were told that they would see faces expressing fear, happy, and anger, or hear short vocalizations that would convey the same three emotions. Two blocks of social stimuli (all faces or vocalizations) were presented. Within blocks, the stimuli were randomized with respect to affective valence. Stimulus blocks were counterbalanced within experimental groups.

For the active task, participants were similarly given instructions on a presentation screen, which was also read aloud by he experimenter. Participants were informed that they would see faces or hear vocalizations sequentially similar to the passive task, and that they would have to identify the emotion expressed after each presentation. Experimenters notified participants that they would be shown a forced-choice response screen (scary/scared, happy, angry) after each presentation to remind them of the possible options. Moreover, participants engaged in brief practice trials to ensure they understood the directions and the three forced- 
Anxiety and autonomic response to emotion in WS

choice responses. Please see Järvinen et al. (2015) for a more detailed description of the procedures involved in psychophysiological recording.

For analysis, we sampled seven seconds subsequent to stimuli presentation and a threesecond pre-stimulus baseline on a trial-by-trial basis, in order to compute event-related change scores. This approach allowed us to obtain weighted trial-specific percentage variations of autonomic activity, thus minimizing the influence of large-scale tonic fluctuations and assessing small-scale ANS reactivity and sensitivity.

\subsection{Data Analysis}

EDA and ECG were qualitatively inspected for possible artefacts. Moreover, all trials containing outliers, i.e., scores that exceeded 2.5 SDs above or below the individual mean were removed from analyses. Mean inter-beat interval (IBI) was calculated from the raw ECG signal to assess differences in heartbeat across social-affective conditions relative to baseline level. For more detailed information regarding psychophysiological recordings, please see Järvinen et al. (2012; 2015a-d), as ANS analyses were replicated.

Repeated measures analyses of covariance (ANCOVAs) with group (WS, TD) as a between-subjects factor, sensory (face, vocal) and emotion (angry, fearful, happy) as withinsubjects factors, and age as a covariate were conducted to determine group differences in emotion recognition accuracy and autonomic responsivity (EDA, mean IBI). Additionally, repeated measures ANCOVA with BAI subscales as within-subjects factor, group as a fixed

factor and age as the covariate were computed. To assess the error patterns in response to each of the three affective condition (angry, fearful, happy), ANCOVAs were applied with sensory (face, voice) and incorrect response type (e.g., fearful/happy; happy/angry; or fearful/angry) as withinsubject variables; group (WS, TD) as a between-group factor; and age as a covariate. The 
Anxiety and autonomic response to emotion in WS

dependent measure was the frequency of errors committed per condition out of the total errors (i.e., error proportion). For example, for a participant who made a total of 8 errors, of which 4 fearful vocalizations were misidentified as angry, the error proportion would be 0.50 . Finally, Pearson correlational analyses were used to determine associations between EDA and mean IBI to anxiety subscores. Post-hoc Bonferroni t-tests were applied to explore significant effects. Across analyses, alpha value was set at 0.05 . It should be noted that the analyses with anxiety measures solely included data from 20 participants with WS and 16 TD individuals, given the aforementioned missing BAI data (see Table 1).

\section{Results}

\subsection{Group differences in anxiety and emotion recognition}

Table 1 outlines the average BAI scores, mean EDA change scores, mean IBI, and the proportion of trials correct on the emotion recognition tasks across social-affective conditions. Individuals with WS endorsed higher anxiety than $\operatorname{TD}$ group $\left(F(1,31)=4.44, p=.04, \eta^{2}=0.13\right)$. No other effects or interactions were found.

A main effect of Emotion was found $\left(F(2,70)=3.29, p=0.04, \eta^{2}=0.09\right)$. Happy expressions $(M=0.99)$ were identified correctly at a greater rate than anger $(M=0.68)$ and fear $(\mathrm{M}=0.93)$, and fearful stimuli were labeled correctly more often than angry targets (ts $>3.71$, ps $<$ 0.001). Interactions between Group x Sensory $\left(F(2,70)=4.62, p=0.04, \eta^{2}=0.11\right)$ and Group $x$ Emotion also emerged $\left(\mathrm{F}(2,70)=4.35, p=0.02, \eta^{2}=0.11\right)$. For the Group x Sensory interaction, post-hoc analyses indicated that WS and TD groups recognized emotions in faces more accurately than in vocal stimuli (WS: $M_{\text {Face }}=0.94, M_{\text {Vocal }}=0.73 ;$ TD: $M_{\text {Face }}=0.98, M_{\text {Vocal }}=0.85$; ts $>4.57$, ps $<0.001)$. Those with WS showed less accuracy identifying affect in vocalizations compared to TD participants $(\mathrm{t}(36)=4.50, p<0.001)$, whereas performance for faces did not 
Anxiety and autonomic response to emotion in WS

reach significance. In regards to Group x Emotion interaction, within those with WS, anger was identified in stimuli less accurately than fear, which in turn yielded poorer performance than happy $\left(M_{\text {Anger }}=0.61, M_{\text {Happy }}=0.99, M_{\text {Fear }}=0.91 ;\right.$ ts $\left.>3.44, p s<0.001\right)$. TD participants similarly identified anger less accurately than fear and happy $\left(M_{\text {Anger }}=0.78, M_{\text {Happy }}=0.99, M_{\text {Fear }}=0.96\right.$; ts $>$ 5.06, $p s<0.001$ ), although the latter two emotions resulted with comparable performance levels. Across emotions, compared to TD group, those with WS showed poorer recognition of anger solely $(\mathrm{t}(36)=3.80, p<0.001)$.

\subsection{Analysis of error patterns in the emotion recognition task}

For the anger condition, significant main effects of sensory condition $(\mathrm{F}(1,36)=7.79, p=0.008$, $\left.\eta^{2}=0.18\right)$ and incorrect response type were found $\left(\mathrm{F}(1,36)=5.06, p=0.03, \eta^{2}=0.12\right)$. These findings indicate that that vocalizations $(M=0.33)$ were more frequently misidentified relative to faces $(M=0.05)$, and angry stimuli were more often erroneously recognized as fearful $(M=0.33)$ rather than happy $(\mathrm{M}=0.05)$. No significant effects were found in the happy and fearful condition.

\subsection{Group differences in psychophysiological measures}

More positive EDA change reflects greater electrodermal activity to the social-affective stimuli whereas more negative EDA values indicate low sympathetic autonomic arousal. Mean IBI indexes heart beat acceleration or deceleration between baseline to stimulus onset. No main effects or interactions reached significance.

\subsection{Associations between electrodermal responses, mean IBI, and emotion recognition accuracy to social-affective stimuli with anxiety}

Pearson correlations were computed between total raw BAI with physiological measures across the three emotions (angry, fearful, happy). Physiological responses to emotions were 
Anxiety and autonomic response to emotion in WS

examined broadly to minimise the number of statistical tests, thereby reducing the risk of committing Type 1 error. Table 2 outlines the correlations found between anxiety and physiological indices. For individuals with WS, an inverse association was found between EDA change score to angry stimuli and BAI total score but no associations were found between IBI measures and anxiety. Among TD individuals, no significant associations were observed. Similarly, correlational analyses were applied between the affect recognition scores (i.e., proportion of correct trials per emotion) and BAI total scores; however, no significant relationships were found.

\section{[Insert Table 2 here]}

\section{Discussion}

The current study examined the interplay between autonomic reactivity to socialemotional stimuli and anxiety in WS and TD individuals. Four important findings emerged. First, individuals with WS displayed greater anxiety relative to TD participants. Second, as compared to the TD participants, individuals with WS showed a specific difficulty in recognising anger across the social stimuli, while also exhibiting a relatively preserved ability to identify happy emotion across social stimuli, suggesting a perceptual bias toward positively valenced stimuli. Third, in contrast to our hypotheses, no significant group differences were observed in autonomic reactivity to social-affective stimuli. Finally, diminished arousal to angry social stimuli was uniquely related to higher anxiety in WS. Taken together, individuals with WS displayed an abnormal autonomic response to angry stimuli, which in turn may contribute to the intensified experience of anxiety ( $\mathrm{Ng}$ et al., 2014).

Consistent with previous behavioural evidence (Leyfer et al., 2006; Ng et al., 2014; Rodgers et al., 2012; Sarimski, 1997; Woodruff-Borden et al., 2010), individuals with WS 
Anxiety and autonomic response to emotion in WS

exhibited greater anxiety relative to TD individuals. Recent evidence also implicates similarities in neural activity and structural integrity between individuals with WS and anxiety disorders. For example, researchers found pathophysiological abnormalities in the temporal-prefrontal connectivity and resting state electroencephalogram activity in the frontal cortical region with anxiety in WS, similar to individuals that meet the diagnosis of an anxiety disorder $(\mathrm{Ng}$, Fishman, \& Bellugi, 2015; Ng et al., 2015). Moreover, a meta-analytic review of functional magnetic resonance imaging (fMRI) evidence of adults with WS vs. adults with social anxiety disorder (SAD) indicated that both groups share an abnormal recruitment of the limbic system, namely amygdala and parahippocampal gyrus (Binelli et al., 2014). Specifically, while individuals with WS had reduced activation of these regions, participants with SAD had hyperactivation of the limbic area, highlighting commonly affected neural networks that may contribute to the diverging social and clinical presentations. In sum, further investigations with individuals with WS and anxiety disorders will be a potentially valuable avenue to better understand how the implicated genes may have a role in the development of the psychopathology.

Persons with WS showed relatively intact recognition of happy affect as compared to TD group, yet struggled to recognise anger, providing some support that individuals with WS may have a social and perceptual bias towards positive stimuli (e.g., Järvinen et al., 2015; JärvinenPasley et al., 2010; Gagliardi et al., 2003; Lacroix et al., 2009; Plesa Skwerer et al., 2006, 2008). For example, individuals with WS have been documented to approach and attend to positive faces more often compared to TD individuals (Dodd \& Porter, 2010; Frigerio et al., 2006). Moreover, detailed data analyses reported by Järvinen and colleagues (2012) showed that those with WS commonly misidentified negative affective stimuli as positive, indicating that their 
Anxiety and autonomic response to emotion in WS

relative strength in the perception of positive social stimuli may occur at the expense of reduced attention and sensitivity to negative affective stimuli. It is noteworthy, however, that individuals with WS show an opposing behavioural and neural pattern (i.e., hypervigilance) to threatening non-social stimuli (e.g., scenic pictures) (Dodd \& Porter, 2011; Meyer-Lindenberg et al., 2005a). Future psychophysiological investigations will need to continue delineating the extent to anxiety in persons with WS is related to the degree of sensitivity towards non-social versus threatening social information.

It should be noted that although our results are consistent with past postulations of a positive perceptual bias in those with WS, both participant groups performed strongly in this condition, suggesting possible ceiling effect. The current investigation employed basic emotions to assess affect perception and related ANS arousal. However, it is possible that use of other positive affect varying in complexity such as proud, joy, and excited may yield differing accuracy rates, as indicated by relatively strong performance of WS participants in the identification of fearful stimuli relative to the other negative-valenced condition, anger. Moreover, our stimuli were presented in isolated fashion, which may not be ecologically valid. In past investigations, the use of more naturalistic stimuli (e.g., faces in a social scenes rather than static facial expressions; human actors compared to cartoon animations) in research with individuals with neurodevelopmental disorders have elicited more syndrome-specific abnormalities in social-information processing, whereas, social perception tasks with less ecologically valid stimuli appeared to mask these perceptual differences (Hanley, McPhillips, Mulhern, \& Riby, 2012; Riby \& Hancock, 2008, 2009).

Additionally, our findings highlight that those with WS do not show severe deficits in emotion recognition across all negative-valenced emotions. Interestingly, both individuals with 
Anxiety and autonomic response to emotion in WS

WS and TD adults showed a similar pattern of weakness in identifying angry expressions, particularly in vocalizations, relative to fear and happy stimuli; albeit, those with WS performed poorer in this affective condition compared to the TD counterparts. However, those with WS showed comparable performance to TD counterparts in identifying fear across social stimuli. Past research findings have supported the postulation that typically developing adults show a 'negativity bias', i.e., a tendency for increased sensitivity towards negative information (Cacioppo, Gardner, \& Berntson, 1999; Öhmann, Lundqvist, \& Esteves, 2001), as this heightened attention towards such information could trigger defensive behaviours and protect oneself. In effect, according to the evolutionary perspective, sensitivity to specific negative emotions has been considered as an adaptive function formed to shield oneself from social dangers (Vaish, Grossman, \& Woodward, 2008). Unexpectedly, both WS and TD participants struggled in identifying anger across vocal and facial targets, yet showed strong capacity to recognize fear in social stimuli. It is possible that these results (i.e., reduced sensitivity to anger and relatively preserved perception of fearful stimuli in those with WS) stem from the limited stimuli and trials administered, and discrepancies in the intensity of the emotion expression across the three affective conditions, which has been shown to influence discriminative sensitivity (Montagne, Kessels, De Haan, \& Perrett, 2007). Furthermore, it should be noted that some research have shown that patients with social anxiety show specific difficulty with recognizing anger relative to other negative emotion (Montagne et al., 2006) and reduced attention to such stimuli (Mansell, Clark, Ehlers, \& Chen, 1999). These authors proffered that reduced sensitivity to angry facial expressions stem from avoidant and maladaptive mechanisms that maintains the clinical disorder. Although past investigators have theorized that the anxiety exhibited by individuals with WS is non-social in nature (Meyer-Lindenber et al., 2005a), further 
Anxiety and autonomic response to emotion in WS

studies examining social anxiety symptomatology with emotion-recognition skills and physiological arousal in those with WS versus TD are warranted.

The relative high sensitivity to fear stimuli in those with WS may also contribute to their unusual social behaviours. Although sensitivity to stimuli signalling threat (e.g., anger) may aide in protecting oneself from danger, increased recognition accuracy to facial expressions of fear has been linked to more prosocial gestures (Marsh et al., 2007). In contrast, individuals with antisocial tendencies demonstrate specific deficits in identifying fear in social stimuli (Marsh \& Blair, 2006). It was contended that expressions of fear serve as distress cues for the expresser, triggering more empathetic behaviours from others. Given the prosocial nature of those with WS (Jones et al., 2001), it is possible that their relatively preserved capacity to recognize fear in social stimuli combined with their poor inhibitory skills (Mobbs et al., 2007) contribute their unusual social profile (e.g., high empathetic concern towards others, inclination to approach others). Likewise, those with WS may reinforced for their prosocial behaviours, which in turn could also foster stronger perceptual sensitivity to fear stimuli.

Importantly, both participant groups exhibited similar autonomic responsivity to socialaffective stimuli, which contrasts with prior investigations (Järvinen-Pasley et al., 2013, 2015; Plesa Skwerer et al., 2009). Interestingly, however, 11 participants with WS scored within the mild to minimal anxiety range on the BAI, suggesting that the current sample may have been associated with an atypically low anxiety. Therefore, it is possible that the similar autonomic responsivity between participant groups at least partially stemmed from the fact that neither was associated with elevated anxiety symptomatology. Typically, elevations in EDA are elicited by stimuli evoking threat (Bradley et al., 2001) or threat appraisal, which may be an adaptive feature to avoid social dangers (Wessing et al., 2011). While individuals with WS typically approach 
Anxiety and autonomic response to emotion in WS

strangers at a significantly greater frequency than TD counterparts (see Järvinen et al., 2013, for a review), empirical evidence has indicated that the increased appetitive social drive (e.g., approach behaviours, prosocial tendencies) may be linked to frontostriatal dysfunction and impaired executive functioning, e.g., poor inhibitory control and planning (Little et al., 2013; Mobbs et al., 2007; Rhodes et al., 2010). Thus, it is also plausible that individuals with WS with low anxiety may exhibit normative autonomic functioning to social stimuli; however, poor impulse control results in their unusual hypersocial behaviors.

A key finding from the present study is the association between reduced arousal to angry stimuli and increased anxiety symptomatology in individuals with WS. Early postulation by Fowles $(1980 ; 1983)$ linked elevated EDA with anxiety and inhibition. It was suggested that increases in EDA in the face of threatening stimuli (e.g., punishment scenarios) are associated with activation of behavioural inhibition system (Fowles, 1980), which represents a neurobiological marker of anxiety (Gray, 1982). Indeed, in the normative developmental literature, reduced sympathetic activity (e.g., electrodermal hyporeactivity) has been associated with reduced anxiety (Clements \& Turpin, 1995) (for a review see Friedman, 2007); yet in our study, participants with WS exhibited the opposite pattern, reflecting disorganisation of the ANS system and poor stress regulation.

The observed inverse relationship between anxiety and electrodermal responses to social presentations of anger in individuals with WS may contribute to their unusual social phenotype. Reduced sympathetic autonomic reactivity to angry social information may reflect weak internal signals to attend to these stimuli, resulting in low physiological anxiety and more confidence to approach persons exhibiting such hostile/aggressive cues, which in turn subjects the individuals with WS to precarious situations. As individuals with WS increasingly encounter social 
Anxiety and autonomic response to emotion in WS

experiences in which they approach others in inappropriate situations, resulting in negative interpersonal interactions, this may contribute to elevated overall anxiety in real life settings in such persons, as indexed by the BAI. Thus, the observed relationship between physiological responses and anxiety in individuals with WS may also reflect a function of conditioned responses over time. Importantly, recent evidence on adolescents and adults with WS show that individual differences in behavioural anxiety are linked to unique face gaze patterns (i.e., greater attention to the mouth and reduced fixation to the eye region of fearful faces) and to compromised social functioning, suggesting that the social behavioural and anxiety characteristics in WS may not be fully dissociable (Kirk et al., 2013). To this end, subsequent research should further explore whether social behaviours may act as a moderator between autonomic reactivity to affective stimuli and anxiety in WS.

Additionally, it is also possible that the overfriendly demeanour and increased anxiety/fears associated with WS are moderated by the limbic system, a network dedicated to emotion processing. Extensive evidence indicates that individuals with WS show atypical amygdala and hippocampal functioning (Hass et al., 2009, 2010; Meyer-Lindenberg et al., 2005; Paul et al., 2009) as well as abnormal structural integrity (Martens et al., 2009; MeyerLindenberg et al., 2005; Reiss et al., 2004) relative to TD and DD individuals (without medical aetiologies). Haas et al. (2010) showed that in individuals with WS, decreased amygdala activation to fearful facial expressions was linked to an elevated tendency to approach strangers. In a similar vein, hyperactivation of the amygdala to positive social stimuli was observed in WS (Haas et al., 2009). Thus, it is likely that functions of the limbic system are affected in persons with WS, thereby impacting bottom-up mechanisms of emotion regulation (i.e., perception and experience of emotions). 
Anxiety and autonomic response to emotion in WS

Likewise, the differential relationships observed between sympathetic autonomic arousal to angry stimuli and anxiety across experimental groups may also stem from differences in the up-regulation of autonomic control. Cognitive control of physiological arousal (e.g., during biofeedback and relaxation exercises) has been linked to the activation of the anterior cingulate cortex, globus pallidum, and parietal cortex (Critchey et al., 2001); thus, these neural substrates play a central role in autonomic regulation. Yet, for individuals with WS, frontostriatal circuits, including the anterior cingulate cortex, are less engaged during response inhibition tasks as compared to TD individuals, suggesting abnormal functioning of the neural circuitry necessary for behavioural inhibition/regulation in WS (Mobbs et al., 2007). This may in turn sub-serve poor autonomic control related to increased stress/anxiety. Additionally, there is supporting evidence showing that individuals with WS have an impoverished insula (i.e., reduced whitematter integrity of the uncinate fasciculus bridging frontal cortical region and the insula), and that increased volume and regional cerebral blood flow of the insula is positively associated with their levels of anxiety, hypersociability, and empathic personality (Jabbi et al., 2012). The insula serves as nexus for interoceptive and emotional information to yield subjective feelings (Craig, 2009; Critchley et al., 2004). Thus, in the context of the current study, it is possible that individuals with WS demonstrate diminished sensitivity to internal bodily cues (e.g., visceral awareness) in response to social-affective stimuli, which in turn influences their ability to apply coping strategies to manage negative emotional state. In effect, the observed atypical associations between psychophysiological responsivity and anxiety in those with WS might stem from dysregulated top-down autonomic and behavioural regulation.

The lack of associations between anxiety with psychophysiological measures to emotional faces and voices among TD individuals may reflect that autonomic reactivity alone is 
Anxiety and autonomic response to emotion in WS

not sufficient to explain psychopathology in normative development. Given that previous evidence suggests that subjective feelings stem from a complex interplay of cognitive processes, emotional awareness, interoceptive awareness, and behavioural responses (Craig, 2009), it is possible psychophysiological responsivity does not directly affect anxiety, and rather, the other noted features mediate the association. Additionally, individual differences such as temperament features (e.g., internalising vs. externalising characteristics; behavioural inhibition vs. activation) further interact with autonomic functioning through development to introduce risks for psychopathology (Beauchaine, 2001; Pérez-Edgar \& Fox, 2005; Vasey \& Dadds, 2001). In order to better understand the neurobehavioural profile associated with anxiety under normative development, it is likely that other factors including temperament, approach/avoidant tendencies, behavioural response to threat-related cues, and recent stressors will need to be considered in conjunction with psychophysiological measures. As such, it is important to highlight that continued integration of multiple methods (e.g., neural, physiological, emotional, temperamentrelated and cognitive measures) will be necessary to provide a more cogent illustration of the complex links between genetic, neural, and behavioural systems that contribute to anxiety in those under normative development versus those with WS.

Limitations of the current study should be considered in the design of future research to better characterize associations between psychophysiological arousal, social-emotion perception, anxiety and social approach behaviours in WS. The current study utilized several statistical tests to examine group differences autonomic responsivity across emotional valence and sensory modalities, which increases the concerns of low statistical power when considering the limited sample sizes. Increasing participant recruitment would be necessary to reduce chances of detecting false positive results. In a similar vein, the inclusion of either mental age matched or a 
Anxiety and autonomic response to emotion in WS

comparison group with DD or intellectual disabilities (ID)(without medical aetiologies) would be an important step towards characterizing features of physiological responsivity to social stimuli that are specific to WS. Notably, the inclusion of an ID comparison group would provide better delineation of emotion recognition patterns and ANS responsivity that are specific to the WS genetic profile versus their cognitive functioning. For example, individuals with WS and ID both show deficits in emotion-recognition, particularly of negative-valenced stimuli (Plesa-Skwerer et al., 2006), and those with WS versus DS have some similarities in approach ratings in response to affective facial stimuli (Porter et al., 2007). As such, our results need to be considered with caution as the differences in behavioural and physiological responsivity between WS and TD adults may be attributed to intellectual functioning rather than genetic profile alone.

Additionally, our inventories were completed by caregivers for the WS group, but, selfreported in the TD group. The inclusion of multiple informants (e.g., self, caregiver, peer) would provide a more cohesive illustration of anxiety across groups, as individuals may be selectively more anxious in specific contexts (e.g., school versus school) and self versus other informants may show different reporting biases and disagreement. For example, Dykens et al. (2003) showed that frequency and intensity of anxiety and phobic symptoms differed when individuals with WS self-reported relative to a caregiver-report. Furthermore, other research have suggested that self-reports may be particularly critical in identifying internalizing experiences (e.g., stress, affective symptoms, subjective feelings) among those with ID, as these factors may not be observable or clearly detected by caregivers or other providers (Lunsky \& Bramston, 2006; Mileviciute \& Hartley, 2015). As such, stronger efforts to integrate self-report of individuals with ID in clinical research could be made to ensure construct validity and appropriate interventions (for examples of recent empirical and clinical approaches, see Patterson, 2012). 
Anxiety and autonomic response to emotion in WS

Finally, as noted above, our study yielded high emotion recognition accuracy in our happy condition, future research may want to provide more challenging affect identification task to better distinguish autonomic indices related to processing of positive-valenced emotions.

In conclusion, the current study provides a preliminary step toward understanding the links between autonomic sensitivity toward social-affective stimuli and anxiety in WS. Namely, unique to individuals with WS, greater anxiety was associated with diminished autonomic arousal to angry social stimuli. These findings provide initial clues linking the social and anxiety phenotypes of WS, suggesting that aberrations in the ANS that have been shown to underlie anomalous emotion perception (Järvinen et al., 2012) and social dysfunction (Järvinen et al., 2015) may also be linked to increased anxiety ( $\mathrm{Ng}$ et al., 2014) in this population. 
Anxiety and autonomic response to emotion in WS

\section{Funding}

This study was supported by the National Institutes of Health [NICHD 0331133, NINDS 22343) and The Oak Tree Philanthropic Foundation awarded to the second author. Additionally, this research was supported the National Science Foundation Graduate Research Fellowship Program (grant 00039202) as part of the first author's training. 
Anxiety and autonomic response to emotion in WS

\section{References}

Adolphs, R. (2003). Cognitive neuroscience of human social behavior. Nature Reviews Neuroscience, $\underline{4}, 165-178$.

Beauchaine, T. (2001). Vagal tone, development, and Gray's motivational theory: Toward an integrated model of autonomic nervous system functioning in psychopathology. Development and Psychopathology, 13, 183-214.

Beck, A. T., Epstein, N., Brown, G., \& Steer, R. A. (1988). An inventory for measuring clinical anxiety: psychometric properties. Journal of Consulting and Clinical Psychology, 56, 893-897.

Bellugi, U., Lichtenberger, L., Jones, W., Lai, Z., \& St George, M. (2000). I. The neurocognitive profile of Williams Syndrome: a complex pattern of strengths and weaknesses. Journal of Cognitive Neuroscience, 12, 7-29.

Bellugi, U., Lichtenberger, L., Mills, D., Galaburda, A., \& Korenberg, J. R. (1999). Bridging cognition, the brain and molecular genetics: evidence from Williams syndrome. Trends in Neurosciences, 22, 197-207.

Bernston, G. G., Quigley K. S., \& Lozano D. (2005). Cardiovascular psychophysiology. In J.T. Cacioppo, L.G. Tassinary, \& G.G. Bernston, Handbook of Psychophysiology (3rd Ed., pp. 182-210). Cambridge: MIT Press.

Binelli, C., Subirà, S., Batalla, A., Muñiz, A., Sugranyés, G., Crippa, J. A., Farré, M., PérezJurado, L., \& Martín-Santos, R. (2014). Common and distinct neural correlates of facial emotion processing in social anxiety disorder and Williams syndrome: A systematic review and voxel-based meta-analysis of functional resonance imaging studies. Neuropsychologia, 64, 205-217. 
Anxiety and autonomic response to emotion in WS

Bradley, M. M., Codispoti, M., Cuthbert, B. N., \& Lang, P. J. (2001). Emotion and motivation I: defensive and appetitive reactions in picture processing. Emotion, 1, pp. 276-298.

Cacioppo, J.T., \& Bernston, G.G. (1999). The affect system: architecture and operating characteristics. Current Directions in Psychological Science, 8, 133-137.

Clements, K., \& Turpin, G. (1995). Effects of feedback and task difficulty on electrodermal activity and heart rate: An examination of Fowles' three arousal model. Journal of Psychophysiology, 9, 231-242.

Cohen, S., Masyn, K., Mastergeorge, A., \& Hessl, D. (2013). Psychophysiological responses to emotional stimuli in children and adolescents with autism and fragile X syndrome. Journal of Clinical Child \& Adolescent Psychology, 44,250-263.

Craig, A. D. (2009). How do you feel - now? The anterior insula and human awareness. Nature Reviews Neuroscience, 10, 50-70.

Critchley, H. D., Melmed, R. N., Featherstone, E., Mathias, C. J., \& Dolan, R. J. 2001. Brain activity during biofeedback relaxation: A functional neuroimaging investigation. Brain. 124(5), pp. 1003-1012.

Critchley, H. D., Wiens, S., Rotshtein, P., Ohman, A., \& Dolan, R. J. (2004). Neural systems supporting interoceptive awareness. Nature Neuroscience, 7,189-195.

Dennis, M., Francis, D. J., Cirino, P. T., Schachar, R., Barnes, M. A., \& Fletcher, J. M. 2009. Why IQ is not a covariate in cognitive studies of neurodevelopmental disorders. Journal of the International Neuropsychological Society. 15, pp. 331-343.

Dimitropoulos, A., Ho, A. Y., Klaiman, C., Koenig, K., \& Schultz, R. T. (2009). A comparison of behavioral and emotional characteristics in children with autism, Prader-Willi 
Anxiety and autonomic response to emotion in WS

syndrome, and Williams syndrome. Journal of Mental Health Research in Intellectual Disabilities. 2, 220-243.

Dodd, H. F., \& Porter, M. A. (2010). I see happy people: attention bias towards happy but not angry facial expressions in Williams syndrome. Cognitive Neuropsychiatry, 15, 549-567.

Dodd, H. F., \& Porter, M. A. (2011). There's that scary picture: Attention bias to threatening scenes in Williams syndrome. Neuropsychologia, 49, 247-253.

Dodd, H. F., Schniering, C. A., \& Porter, M. A. (2009). Beyond behavior: Is social anxiety low in Williams syndrome? Journal of Autism and Developmental Disorders, 39,1673-1681.

Doyle, T. F., Bellugi, U., Korenberg, J. R., \& Graham, J. (2004). "Everybody in the world is my friend" hypersociability in young children with Williams syndrome. American Journal of Medical Genetics Part A, 124,263-273.

Dykens, E. M. (2003). Anxiety, fears, and phobias in persons with Williams syndrome. Developmental Neuropsychology, 23, 291-316.

Dykens, E. M., Rosner, B. A., Ly, T., \& Sagun, J. (2005). Music and anxiety in Williams syndrome: a harmonious or discordant relationship? American Journal on Mental Retardation, 110, 346-358.

Ewart, A. K., Morris, C. A., Atkinson, D., Jin, W., Sternes, K., Spallone, P., Dean Stock, A., Leppert, M., \& Keating, M. T. (1993). Hemizygosity at the elastin locus in a developmental disorder, Williams syndrome. Nature Genetics, 5, 11-16.

Fowles, D. C. (1980). The three arousal model: Implications of Gray's two- factor learning theory for heart rate, electrodermal activity, and psychopathy. Psychophysiology, 17, 87104. 
Anxiety and autonomic response to emotion in WS

Fowles, D. C. (1983). Motivation effects on heart rate and electrodermal activity: Implications for research on personality and psychopathology. Journal of Research in Personality, 17, 48-71.

Friedman, B. H. (2007). An autonomic flexibility-neurovisceral integration model of anxiety and cardiac vagal tone. Biological psychology, 74, 185-199.

Frigerio, E., Burt, D. M., Gagliardi, C., Cioffi, G., Martelli, S., Perrett, D. I., \& Borgatti, R. (2006). Is everybody always my friend? Perception of approachability in Williams syndrome. Neuropsychologia, 44,254-259.

Gagliardi, C., Frigerio, E., Burt, D. M., Cazzaniga, I., Perrett, D. I., \& Borgatti, R. (2003). Facial expression recognition in Williams syndrome. Neuropsychologia, 41,733-738.

Gray, J. A. (1982). On mapping anxiety. Behavioral and Brain Sciences, 5, 506-534.

Haas, B. W., Hoeft, F., Searcy, Y. M., Mills, D., Bellugi, U., \& Reiss, A. (2010). Individual differences in social behavior predict amygdala response to fearful facial expressions in Williams syndrome. Neuropsychologia, 48,1283-1288.

Haas, B. W., Mills, D., Yam, A., Hoeft, F., Bellugi, U., \& Reiss, A. (2009). Genetic influences on sociability: heightened amygdala reactivity and event-related responses to positive social stimuli in Williams syndrome. Journal of Neuroscience, 29, 1132-1139.

Haas, B. W., \& Reiss, A. L. (2012). Social brain development in Williams syndrome: the current status and directions for future research. Frontiers in Psychology, 3, 186.

Hanley, M., McPhillips, M., Mulhern, G., \& Riby, D.M. (2012). Spontaneous attention to faces in Asperger syndrome using ecologically valid static stimuli. Autism, 17(6), 754-761. 
Anxiety and autonomic response to emotion in WS

Herman, J. P., Ostrander, M. M., Mueller, N. K., \& Figueiredo, H. (2005). Limbic system mechanisms of stress regulation: hypothalamo-pituitary-adrenocortical axis. Progress in Neuro-Psychopharmacology and Biological Psychiatry, 29, 1201-1213.

Hillier, L.W., Fulton, R.S., Fultin, L.A., Graves, T.A., Pepin, K.H.,... Wilson R.K. (2003). The DNA sequence of human chromosome 7. Nature, 424, 157-164.

Jabbi, M., Kippenhan, J. S., Kohn, P., Marenco, S., Mervis, C. B., Morris, C. A., MeyerLindenberg, A., \& Berman, K. F. (2012). The Williams syndrome chromosome 7q11. 23 hemideletion confers hypersocial, anxious personality coupled with altered insula structure and function. Proceedings of the National Academy of Sciences, 109, E860E866.

Järvinen, A. M., \& Bellugi, U. (2013). What does Williams syndrome reveal about the determinants of social behavior? Frontiers in Human Neuroscience, 7, 1-6.

Järvinen, A., Dering, B., Neumann, D., Ng, R., Crivelli, D., Grichanik, M., Korenberg, J. R., \& Bellugi, U. (2012). Sensitivity of the autonomic nervous system to visual and auditory affect across social and non-social domains in Williams syndrome. Frontiers in Psychology, 3, 1-16.

Järvinen, A., Ng, R., \& Bellugi, U. (2015). Autonomic response to approachability characteristics, approach behavior, and social functioning in Williams syndrome. Neuropsychologia, 78, 159-170.

Järvinen, A., Ng, R., Crivelli, D., Arnold, A., Woo-VonHoogenstyn, N., \& Bellugi, U. (2015). Relations between social-perceptual ability in multi- and unisensory contexts, autonomic reactivity, and social functioning in individuals with Williams syndrome. Neuropsychologia, 73, 127-140. 
Anxiety and autonomic response to emotion in WS

Järvinen, A., Ng, R., Crivelli, D., Neumann, D., Arnold, A., Woo-VonHoogenstyn, N., Lai, P., Trauner, D., \& Bellugi, U. (2016). Social functioning and autonomic nervous system sensitivity across vocal and musical emotion in Williams syndrome and autism spectrum disorder. Developmental Psychobiology, 58, 17-26.

Järvinen, A., Ng, R., Crivelli, D., Neumann, D., Grichanik, M., Arnold, A., Lai, P., Trauner, D., \& Bellugi, U. (2015). Patterns of sensitivity to emotion in children with Williams syndrome and autism: Indices of autonomic nervous system reactivity and social functioning. Journal of Autism and Developmental Disorders, 45, 2594-2612.

Järvinen, A., Korenberg, J. R., \& Bellugi, U. (2013). The social phenotype of Williams syndrome. Current Opinion in Neurobiology, 23, 414-422.

Järvinen-Pasley, A., Adolphs, R., Yam, A., Hill, K. J., Grichanik, M., Reilly, J., Mills, D., Reiss, A.L., Korenberg, J.R., \& Bellugi, U. (2010a). Affiliative behavior in Williams syndrome: social perception and real-life social behavior. Neuropsychologia, 48, 2110-2119.

Järvinen-Pasley, A., Bellugi, U., Reilly, J., Mills, D. L., Galaburda, A., Reiss, A. L., \& Korenberg, J. R. (2008). Defining the social phenotype in Williams syndrome: a model for linking gene, the brain, and behavior. Development and Psychopathology, 20,1-35. Järvinen-Pasley, A., Pollak, S. D., Yam, A., Hill, K. J., Grichanik, M., Mills, D., Reiss, A.L., Korenberg, J. R., \& Bellugi, U. (2010b). Atypical hemispheric asymmetry in the perception of negative human vocalizations in individuals with Williams syndrome. Neuropsychologia, 48, 1047-1052.

Jones, W., Bellugi, U., Lai, Z., Chiles, M., Reilly, J., Lincoln, A., \& Adolphs, R. (2000). II. Hypersociability in Williams syndrome. Journal of Cognitive Neuroscience, 12, 30-46. 
Anxiety and autonomic response to emotion in WS

Kirk, H. E., Hocking, D. R., Riby, D. M., \& Cornish, K. M. (2013). Linking social behaviour and anxiety to attention to emotional faces in Williams syndrome. Research in Developmental Disabilities, 34, 4608-4616.

Klein-Tasman, B. P., \& Mervis, C. B. (2003). Distinctive personality characteristics of 8-, 9-, and 10-year-olds with Williams syndrome. Developmental Neuropsychology, 23, 269290.

Lacroix, A., Michèle G., Bernadette R., \& Judy. R. (2009). Recognition of emotional and nonemotional facial expressions: A comparison between Williams syndrome and autism. Research in Developmental Disabilities, 30, 976-985.

Leyfer, O. T., Woodruff-Borden, J., Klein-Tasman, B. P., Fricke, J. S., \& Mervis, C. B. (2006). Prevalence of psychiatric disorders in 4 to 16-year-olds with Williams syndrome. American Journal of Medical Genetics Part B: Neuropsychiatric Genetics, 141, 615-622.

Leyfer, O., Woodruff-Borden, J., \& Mervis, C. B. (2009). Anxiety disorders in children with Williams syndrome, their mothers, and their siblings: Implications for the etiology of anxiety disorders. Journal of Neurodevelopmental Disorders, 1, 4-14.

Lindsay, W.R., \& Skene, D.D. (2007). The Beck Depression Inventory II and the Beck Anxiety Inventory in people with intellectual disabilities: factor analyses and group data. Journal of Applied Research in Intellectual Disabilities, 20(5), 401-408.

Little, K., Riby, D. M., Janes, E., Clark, F., Fleck, R., \& Rodgers, J. (2013). Heterogeneity of social approach behaviour in Williams syndrome: the role of response inhibition. Research in Developmental Disabilities, 34, 959-967. 
Anxiety and autonomic response to emotion in WS

Losh, M., Bellugi, U., \& Anderson, J. D. (2001). Narrative as a social engagement tool: The excessive use of evaluation in narratives from children with Williams syndrome. Narrative Inquiry, 10, 265-290.

Lunsky, Y., \& Bramston, P. (2006). A preliminary study of perceived stress in adults with intellectual disabilities according to self-report and informant ratings. Journal of Intellectual and Developmental Disability, 31(1), 20-27.

Mansell, W., Clark, D.M., Ehlers, A., \& Chen, Y. (1999). Social anxiety and attention away from emotional faces. Cognition and Emotion, 12(6), 673-690.

Marsh, A.A., \& Blair, R.J.R. (2008). Deficits in facial affect recognition among antisocial populations: a meta-analysis. Neuroscience and Biobehavioral Reviews, 32(3), 454-465.

Marsh, A.A., Kozak, M.N., \& Ambady, N. (2007). Accurate identification of fear facial expressions predicts prosocial behavior. Emotion, 7(2), 239-251.

Martens, M. A., Wilson, S. J., Dudgeon, P., \& Reutens, D. C. (2009). Approachability and the amygdala: insights from Williams syndrome. Neuropsychologia, 47,2446-2453.

Meda, S. A., Pryweller, J. R., \& Thornton-Wells, T. A. (2012). Regional brain differences in cortical thickness, surface area and subcortical volume in individuals with Williams syndrome. PLoS One, 7, e31913.

Mervis, C. B., Morris, C. A., Klein-Tasman, B. P., Bertrand, J., Kwitny, S., Appelbaum, L. G., \& Rice, C. E. (2003). Attentional characteristics of infants and toddlers with Williams syndrome during triadic interactions. Developmental Neuropsychology, 23, pp. 243-268 Meyer-Lindenberg, A., Hariri, A. R., Munoz, K. E., Mervis, C. B., Mattay, V. S., Morris, C. A., \& Berman, K. F. (2005a). Neural correlates of genetically abnormal social cognition in Williams syndrome. Nature Neuroscience, 8, 991-993. 
Anxiety and autonomic response to emotion in WS

Meyer-Lindenberg, A., Mervis, C.B., Sarpal, D., Koch, P., Steele, S., Kohn, P., Marenco, S., Morris, C.A., Das, S., Kippenhan, S., \& Mattay, V.S. (2005b). Functional, structural, and metabolic abnormalities of the hippocampal formation in Williams syndrome. Journal of Clinical Investigation, 115,1888.

Mileviciute, I., \& Hartley, S.L. (2015). Self-reported versus informant-reported depressive symptoms in adults with mild intellectual disability. Journal of Intellectual Disability Research, 59(2), 158-169.

Mobbs, D., Eckert, M. A., Mills, D., Korenberg, J., Bellugi, U., Galaburda, A. M., \& Reiss, A. L. (2007). Frontostriatal dysfunction during response inhibition in Williams syndrome. Biological Psychiatry, 62, 256-261.

Montagne, B., Kessels, R.P.C., De Haan, E.H.F., \& Perrett, D.I. (2007). The emotion recognition task: a paradigm to measure the perception of facial emotion expressions at different intensities. Perceptual and Motor Skills, 104(2), 589-598.

Montagne, B., Schutters, S., Westenberg, H.G.M., van Honk, J., Kessels, R.P.C., \& De Haan, E.H.F. (2006). Reduced sensitivity in the recognition of anger and disgust in social anxiety disorder. Cognitive Neuropsychiatry, 11(4), 389-401.

Morris, C. A. (2010). The behavioral phenotype of Williams syndrome: a recognizable pattern of neurodevelopment. American Journal of Medical Genetics Part C: Seminars in Medical Genetics, 154, 427-431.

Morris, C. A., \& Mervis, C. B. (2000). Williams syndrome and related disorders. Annual Review of Genomics and Human Genetics, 1, 461-484. 
Anxiety and autonomic response to emotion in WS

Ng, R., Brown, T.T., Järvinen, A., Erhart, M., Korenberg, J.R., Bellugi, U., \& Halgren, E. (2016). Structural integrity of the limbic-prefrontal connection: Neuropathological correlates of anxiety in Williams syndrome. Social Neuroscience, 11, 187-192.

Ng, R., Järvinen, A., \& Bellugi, U. (2014). Characterizing associations and dissociations between anxiety, social, and cognitive phenotypes of Williams syndrome. Research in Developmental Disabilities, 35, 2403-2415.

Öhmann, A., Lundqvist, D., \& Esteves, F. (2001). The face in the crowd revisited: a threat advantage with schematic stimuli. Journal of Personality and Social Psychology, 80, 381-391.

Osman, A., Kopper, B. A., Barrios, F. X., Osman, J. R., \& Wade, T. (1997). The Beck Anxiety Inventory: Reexamination of factor structure and psychometric properties. Journal of Clinical Psychology, 53, 7-14.

Paul, B. M., Snyder, A. Z., Haist, F., Raichle, M. E., Bellugi, U., \& Stiles, J. (2009). Amygdala response to faces parallels social behavior in Williams syndrome. Social Cognitive and Affective Neuroscience, 4, 278-285.

Patterson, R. (2012). Strategies to incorporate the voices of people with significant disabilities in UCEDD information gathering and operations. Retrieved from www.aucd.org/docs/urc/AUCD_StrategiesReport_Final.pdf

Pérez-Edgar, K., \& Fox, N. A. (2005). Temperament and anxiety disorders. Child and Adolescent Psychiatric Clinics of North America, 14, 681-706.

Pérez-García, D., Granero, R., Gallastegui, F., Pérez-Jurado, L. A., \& Brun-Gasca, C. (2011). Behavioral features of Williams Beuren syndrome compared to Fragile X syndrome and 
Anxiety and autonomic response to emotion in WS

subjects with intellectual disability without defined etiology. Research in Developmental Disabilities, 32, 643-652.

Pfaff, D. W., Kieffer, B. L., \& Swanson, L. W. (2008). Mechanisms for the Regulation of State Changes in the Central Nervous System. Annals of the New York Academy of Sciences, $1129,1-7$.

Pinheiro, J., Bates, D., DebRoy, S., \& Sarkar, D. (2013). The R Development Core Team. NLME: linear and nonlinear mixed effects models, 3-1.

Plesa Skwerer, D., Borum, L., Verbalis, A., Schofield, C., Crawford, N., Ciciolla, L., \& TagerFlusberg, H. (2009). Autonomic responses to dynamic displays of facial expressions in adolescents and adults with Williams syndrome. Social Cognitive and Affective Neuroscience, 4, 93-100.

Plesa Skwerer, D., Verbalis, A., Schofield, C., Faja, S., \& Tager-Flusberg, H. (2006). Socialperceptual abilities in adolescents and adults with Williams syndrome. Cognitive Neuropsychology, 23, 338-349.

Porter, M. A., Coltheart, M., \& Langdon, R. (2007). The neuropsychological basis of hypersociability in Williams and Down syndrome. Neuropsychologia, 45, 2839-2849.

R Development Core Team (2008). Vienna, Austria: R Foundation for Statistical Computing. R: A language and environment for statistical computing.

Reilly, J., Losh, M., Bellugi, U., \& Wulfeck, B. (2004). “Frog, where are you?” Narratives in children with specific language impairment, early focal brain injury, and Williams syndrome. Brain and Language, 88, 229-247. 
Anxiety and autonomic response to emotion in WS

Reiss, A.L., Eckert, M.A., Rose, F., Karchemskiy, A., Kesler, S., Chang, M., Reynolds, M.F., Kwon, H., \& Galaburda, A. (2004). An experiment of nature: Brain anatomy parallels cognition and behavior in Williams syndrome. Journal of Neuroscience, 24, 5009-5015.

Rhodes, S. M., Riby, D. M., Park, J., Fraser, E., \& Campbell, L. E. (2010). Executive neuropsychological functioning in individuals with Williams syndrome. Neuropsychologia, 48, 1216-1226.

Riby, D. M., \& Hancock, P. J. (2008). Viewing it differently: Social scene perception in Williams syndrome and autism. Neuropsychologia, 46, 2855-2860.

Riby, D. M., \& Hancock, P. J. (2009). Do faces capture the attention of individuals with Williams syndrome or autism? Evidence from tracking eye movements. Journal of Autism and Developmental Disorders, 39, 421-431.

Riby, D. M., Hanley, M., Kirk, H., Clark, F., Little, K., Fleck, R.,...Rodgers, J. (2014). The interplay between anxiety and social functioning in Williams syndrome. Journal of Autism and Developmental Disorders, 44, 1220-1229.

Riby, D. M., Whittle, L., \& Doherty-Sneddon, G. (2012). Physiological reactivity to faces via live and video-mediated communication in typical and atypical development. Journal of Clinical and Experimental Neuropsychology, 34, 385-395.

Rodgers, J., Riby, D. M., Janes, E., Connolly, B., \& McConachie, H. (2012). Anxiety and repetitive behaviors in autism spectrum disorders and Williams syndrome: A crosssyndrome comparison. Journal of Autism and Developmental Disorders, 42, 175-180.

Santos, A., Silva, C., Rosset, D., \& Deruelle, C. (2010). Just another face in the crowd: Evidence for decreased detection of angry faces in children with Williams syndrome. Neuropsychologia, 48, 1071-1078. 
Anxiety and autonomic response to emotion in WS

Sarimski, K. (1997). Behavioral phenotypes and family stress in three mental retardation syndromes. European Child \& Adolescent Psychiatry, 6, 26-31.

Searcy, Y. M., Lincoln, A. J., Rose, F. E., Klima, E. S., Bavar, N., \& Korenberg, J. R. (2004). The relationship between age and IQ in adults with Williams syndrome. American Journal on Mental Retardation, 109, 231-236.

Thornton-Wells, T. A., Avery, S. N., \& Blackford, J. U. (2011). Using novel control groups to dissect the amygdala's role in Williams Syndrome. Developmental Cognitive Neuroscience, 1, 295-304.

Tottenham, N., Tanaka, J., Leon, A.C., McCarry, T., Nurse, M., Hare, T.A., Marcus, D.J., Westerlund, A., Casey, B.J., \& Nelson, C.A. (2009). The NimStim set of facial expressions: Judgments from untrained research participants. Psychiatry Research, 168, 242-249.

Vaish, A., Grossman, T., \& Woodward, A. (2008). Not all emotions are created equal: the negativity bias in social-emotional development. Psychological Bulletin, 134(3), 383403.

Vasey, Michael W., \& Mark R. Dadds. (2001). The developmental psychopathology of anxiety. New York, NY: Oxford University Press.

Venables, P. H., \& Christie, M. J. (1980). Electrodermal activity. Techniques in Psychophysiology, 74, 3-67.

Wechsler, D. 1997. WAIS-III, Wechsler adult intelligence scale: Administration and scoring manual. San Antonio, TX: Psychological Corporation.

Wechsler, D. 1999. Wechsler abbreviated scale of intelligence. San Antonio, TX: Psychological Corporation. 
Anxiety and autonomic response to emotion in WS

Wessing, I., Rehbein, M. A., Postert, C., Fürniss, T., \& Junghöfer, M. (2013). The neural basis of cognitive change: reappraisal of emotional faces modulates neural source activity in a frontoparietal attention network. Neuroimage, 81, 15-25.

Woodruff-Borden, J., Kistler, D. J., Henderson, D. R., Crawford, N. A., \& Mervis, C. B. (2010). Longitudinal course of anxiety in children and adolescents with Williams syndrome. American Journal of Medical Genetics Part C: Seminars in Medical Genetics, 154, 277290. 
Table 1. Average age and mean scores for standardized event-related electrodermal activity, interbeat interval, emotion recognition accuracy, and the Beck Anxiety Inventory indices (standard deviations in parentheses) for participants with WS and TD.

\begin{tabular}{|c|c|c|}
\hline & WS & TD \\
\hline$N$ & 25 & 16 \\
\hline Age (years) & $33.45(10.07)$ & 25.44(6.77) \\
\hline & range: $19.01-56.9$ & range: $17.4-43.1$ \\
\hline \multicolumn{3}{|l|}{$\begin{array}{l}\text { Standardized event-related electrodermal } \\
\text { activity (EDA) }\end{array}$} \\
\hline Angry vocal & $-0.06(0.34)$ & $0.10(0.69)$ \\
\hline Angry face & $-0.10(0.27)$ & $-0.10(0.21)$ \\
\hline Fearful vocal & $-0.02(0.48)$ & $0.07(0.45)$ \\
\hline Fearful face & $-0.17(0.18)$ & $0.01(0.68)$ \\
\hline Happy vocal & $0.01(0.39)$ & $0.03(0.56)$ \\
\hline Happy face & $-0.06(0.20)$ & $-0.22(0.25)$ \\
\hline \multicolumn{3}{|l|}{ Mean Interbeat Interval (IBI) } \\
\hline Angry vocal & $0.00(0.26)$ & $-0.06(0.53)$ \\
\hline Angry face & $0.01(0.32)$ & $0.10(0.20)$ \\
\hline Fearful vocal & $-0.08(0.35)$ & $0.08(0.50)$ \\
\hline Fearful face & $-0.11(0.30)$ & $0.15(0.43)$ \\
\hline Happy vocal & $-0.17(0.34)$ & $0.06(0.34)$ \\
\hline Happy face & $-0.09(0.32)$ & $0.06(0.48)$ \\
\hline \multicolumn{3}{|l|}{ Emotion Recognition (proportion correct) } \\
\hline Angry vocal & $0.33(0.29)$ & $0.64(0.24)$ \\
\hline Angry face & $0.88(0.15)$ & $0.94(0.10)$ \\
\hline Fearful vocal & $0.86(0.22)$ & $0.94(0.10)$ \\
\hline Fearful face & $0.95(0.09)$ & $0.99(0.04)$ \\
\hline Happy vocal & $0.99(0.03)$ & $0.99(0.04)$ \\
\hline Happy face & $1.00(0.00)$ & $1.00(0.00)$ \\
\hline Beck Anxiety Inventory (BAI; mean ratings) & $\mathrm{n}=20^{*}$ & $\mathrm{n}=14 *$ \\
\hline Neurophysiological symptoms (BAI-N) & $0.25(0.39)$ & $0.11(0.23)$ \\
\hline Subjective symptoms (BAI-S) & $0.48(0.55)$ & $0.29(0.33)$ \\
\hline Panic symptoms (BAI-P) & $0.30(0.55)$ & $0.09(0.16)$ \\
\hline Autonomic symptoms (BAI-A) & $0.43(0.56)$ & $0.32(0.47)$ \\
\hline Total Raw Score & $7.50(8.61)$ & $4.14(4.85)$ \\
\hline
\end{tabular}

\footnotetext{
*Note. Five WS participants and two TD participants were missing BAI data, as the inventory was not returned in the mail.
} 
Table 2. Associations between EDA change scores and BAI Total for participants with WS $(n=20)$ and TD individuals $(n=14)$.

\begin{tabular}{lr}
\hline $\begin{array}{l}\text { EDA change scores } \\
\text { to emotion }\end{array}$ & \multicolumn{1}{c}{ BAI } \\
\hline Williams Syndrome & \\
Anger & $-\mathbf{0 . 5 2}(\mathbf{0 . 0 2})$ \\
Fear & $-0.21(0.38)$ \\
Happy & $-0.09(0.71)$ \\
& \\
Typical Development & \\
Anger & $0.22(0.45)$ \\
Fear & $-0.12(0.69)$ \\
Happy & $-0.29(0.32)$ \\
\hline Note. P-values are in parentheses.
\end{tabular}

